\title{
Terminologie und Klassifikation der Beckenverletzungen
}

\author{
Ulf Culemann, Heinrich Reilmann
}

\section{Zusammenfassung}

Beckenringfrakturen stellen eine häufige Begleitverletzung des Polytraumas dar, aber auch bei isolierten Verletzungen des Beckenringes können für den Patienten signifikante Folgezustände nach Ende der Behandlung aufgrund beckenbedingter Begleitverletzungen verbleiben. Nur eindeutige Kriterien und Definitionen können eine erfolgreiche Diagnostik und Behandlung gewährleisten. Die Basis eines zielorientierten Managements stellen eine gemeinsame Terminologie und Klassifikation der Beckenringverletzung dar. Aufgrund biomechanischer Erkenntnisse wird funktionell der Beckenring in einen vorderen und einen hinteren Abschnitt gegliedert. Des Weiteren wird in „stabile und instabile" Frakturen unterteilt, von denen einfache und „komplexe“ Beckenfrakturen, die sich durch das zusätzliche Weichteiltrauma definieren, getrennt betrachtet werden müssen. Die heute gebräuchliche Klassifikation der Beckenringfraktur nach der AO/ASIF differenziert in Typ-A-, Typ-B- und Typ-C-Frakturen, die entsprechend ihres Stabilitätsgrades eingeteilt werden. Bei Typ-A-Frakturen handelt es sich um stabile Beckenring- frakturen, die sowohl ventrale Ringunterbrechungen durch Schambeinastfrakturen als auch knöcherne Abrissfrakturen und Querfrakturen des Sakrums unterhalb S2 beinhalten können. Bei den Typ-B-Verletzungen liegen partiell stabile Frakturen vor. Die zumeist durch einen Seitanprall verursachte Außen- oder Innenrotation einer Beckenhälfte führt sowohl zu Frakturen im vorderen Beckenringbereich als auch zu einer partiellen Mitverletzung osteoligamentärer Strukturen im hinteren Beckenringbereich. Typ-C-Verletzungen führen aufgrund der massiven Gewalteinwirkung zu Frakturen im vorderen und hinteren Beckenringbereich, es resultiert eine komplette Instabilität mit Verschiebung einer Beckenhälfte nach kranial oder kaudal. Eine einheitliche Beschreibung der Beckenringverletzung in der Diagnostik durch eine gemeinsame „Sprache“ führt zu einer verbindlichen Klassifikation der Fraktur. Durch klare Definitionen begleitender Weichteilverletzungen und der Fraktureinteilung wird konsekutiv das entsprechende Therapieverfahren abgeleitet, insofern werden für die Behandlungsprognose der Verletzung entscheidende Weichen frühzeitig gestellt.

\section{Einleitung}

Terminologie bei Beckenringverletzungen

Bei 3-5\% aller Skelettfrakturen handelt es sich um Beckenfrakturen, die in der Mehrzahl der Fälle durch Hochrasanztraumen, Stürzen aus großer Höhe oder direkten Einklemmungen entstehen $[10,13]$. Trotz dieser zunächst relativ niedrig erscheinenden Inzidenz kommt

OP-JOURNAL 2003; 19: 100-104

(c) Georg Thieme Verlag Stuttgart · New York den Frakturen des Beckens dennoch eine hohe klinische Relevanz zu, da eine Letalität in $7 \%$ der Fälle bei „einfachen“ und in 20\% der Fälle bei „komplexen“ Beckentraumata auftritt $[1,4,10$, 11,13,14]. Dieser deutliche Anstieg der Letalität erklärt sich aus der begleitenden Weichteilverletzung, die das „komplexe“ Beckentrauma definitionsgemäß von der „einfachen“ Beckenverletzung unterscheidet.

Bei „einfachen“ Beckenfrakturen handelt es sich um rein knöcherne Verletzungen des vorderen oder hinteren Beckenringes ohne begleitende Weichteilverletzung. Im Vordergrund der Verletzung steht die Wiederherstellung der osteoligamentären Integrität des Beckens.

Die begleitende Weichteilverletzung bei der komplexen Beckenfraktur führt nicht nur zu einem Anstieg der Letalität, sondern kompliziert auch die Therapie und bestimmt damit die Prognose des Patienten. Eindeutige Definitionen der begleitenden Weichteilverletzungen sollen dazu beitragen, die Schwere einer Beckenfraktur korrekt einschätzen zu können.

\section{Weichteilverletzungen am Becken}

\section{Das Komplextrauma des Beckens}

Eine „komplexe“ Beckenverletzung ist definiert als Beckenringfaktur mit begleitendem peripelvinen Weichteilschaden, d.h. einer zusätzlichen Verletzung von Nerven, Gefäßen, Muskulatur oder den Beckeneingeweiden $[1,13]$.

Durch den zusätzlich zur knöchernen Fraktur entstandenen Gewebeschaden und Blutverlust kann eine komplexe Bekkenfraktur den Schockzustand des Patienten vertiefen, insofern ist eine Einschätzung der begleitenden Blutungsschwere ebenfalls wichtig (Abb.1).

Zur Abschätzung der Blutungsschwere kann nach Trunkey die Blutverlustmenge pro Zeitintervall ( $\mathrm{ml} / \mathrm{min}$ ) oder nach Bone die Gesamtblutverlustmenge ( $\mathrm{ml}$ ) mit entsprechender Einteilung in Schweregrade angegeben werden. Die Anwendung dieser Berechnung ist jedoch klinisch nicht einsatzfähig in der Notfallsituation. Weiterhin besteht eine prädiktive Beziehung zwischen Letalität und Anzahl der verabreichten Erythrozythenkonzentrate. Dies kann nur rückwirkend berechnet werden, „rückwirkend“ bedeutet für den Patienten aber „zu spät“. Andere prädiktive Parameter wie Thrombozytenzahlen, Interleukine oder Laktat 


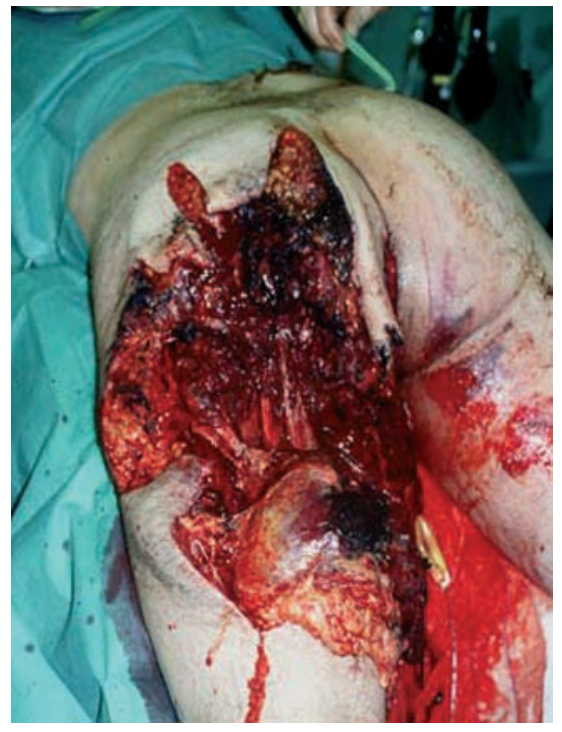

Abb.1 Explosionsverletzung der linken Beckenhälfte mit Komplextrauma des Beckens. Durch die massive Weichteilzerstörung ergibt sich nicht nur ein hoher Blutverlust in der primären Behandlungsphase, sondern auch ein hohes Infektionsrisiko aufgrund der Wundfläche. Die begleitende knöcherne Verletzung kann dabei aufgrund der hohen Energieabsorption der Weichteile vergleichsweise gering ausfallen.

fallen bzw. steigen erst in den ersten Stunden bis Tagen an, auch hier ergibt sich kein direkter Behandlungsgewinn in der ersten Behandlungsphase. Für den klinischen Gebrauch hat sich daher die Abschätzung des Blutverlustes aus dem ersten, direkt bei Aufnahme entnommenen $\mathrm{Hb}-$ Gehalt bewährt. Bei einem Wert unter $8 \mathrm{mg} \%$ kann näherungsweise von einem relevanten Blutverlust mit konsekutiv instabilem Kreislauf ausgegangen werden, der substitutionspflichtig ist [13].

\section{Offene Beckenfrakturen}

Bei „offene Beckenringfrakturen“ kommt es zu knöchernen Durchspießungen der äußeren Haut oder von Hohlorganen des Beckens.

Neben dem initialen Verblutungstod sind die Patienten insbesondere durch die sekundäre Keimbesiedelung gefährdet.

Begleitende Weichteilverletzungen des Perianalbereiches stellen hierfür ein besonders hohes Risiko dar. Durch ein chirurgisch eindeutiges, stufenweises Behandlungskonzept mit frühzeitiger Stabilisierung der Beckenfraktur, großzügiger

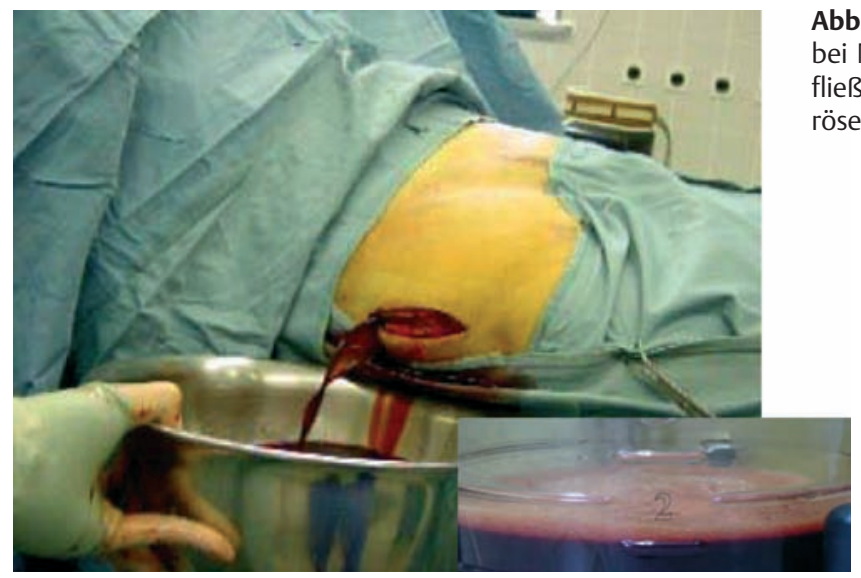

Indikation zur Anlage eines Anus praeter und geplanten second look-Debridements der Weichteile lassen sich Komplikationen wie septische Krankheitsverläufe deutlich reduzieren.

\section{Kompartmentsyndrom des Beckens}

Einfache begleitende Weichteilveränderungen stellen Kontusionsfolgen wie Hämatome und Serome dar, die durch eine rein konservative Behandlung zur Ausheilung gebracht werden. Handelt es sich um ausgedehnte Hämatome mit entsprechend hoher Einblutung in die Muskelfaszienräume des Beckens kann es auch bei fehlenden knöchernen Verletzungen zu einem Kompartmentsyndrom im Bereich der Beckenmuskulatur und hier insbesondere im Gluteal- oder (seltener) im Psoasbereich kommen. Das Kompartment kann durch Druck sekundär zu Nervenschädigungen und Muskelnekrosen mit funktionellen Defiziten führen, insofern ist eine rechtzeitige operative Entlastung notwendig.
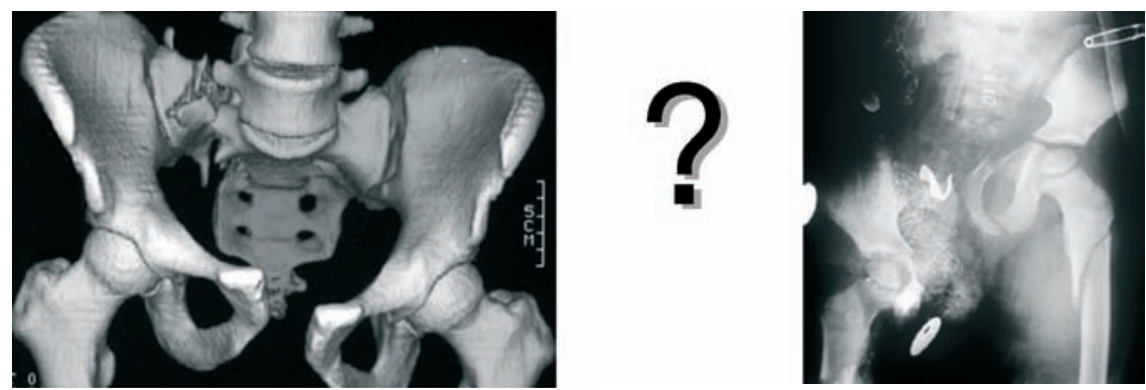

Abb. 3 Beide Röntgenbilder stellen C-Verletzungen des Beckens dar, aber: C- ist nicht gleich C-Verletzung! Das Beispiel links zeigt eine C-1-Verletzung ohne Weichteilschaden (klinisch). Rechts ist eine traumatische Hemipelvektomie im Röntgenbild dargestellt. Die begleitende Weichteilverletzung lässt sich bereits hierdurch erahnen. Bei der Hemipelvektomie handelt es sich um die Maximalvariante einer „komplexen Beckenfraktur“ mit kompletter, neurovaskulärer Abtrennung des rechten Beines. Therapeutisch kann insbesondere aufgrund der begleitenden Nervenausrisse die innerlich bestehende Amputation meist nur noch komplettiert werden, um einen weitergehenden Blutverlust zu vermeiden. 
vom Körperstamm kommt (Abb.3). Die komplette Zerreißung der beinversorgenden Nerven bedeutet dabei selbst bei möglichem Erhalt der Extremität eine Gebrauchsunfähigkeit des Beines, weshalb meist nur eine chirurgische Komplettierung der bereits bestehenden inneren Amputation möglich ist [13].

\section{Knöcherne Verletzungen am Beckenring}

\section{Biomechanische Einteilung des Beckenringes}

Für die Beschreibung einer knöchernen Verletzung des Beckenringes wird zunächst unterschieden in einen vorderen und einen hinteren Abschnitt des Beckenringes. Der vordere Beckenring umfasst dabei den Bereich der Schambeine (oberer und unterer Ast) und des Sitzbeines bis in Höhe des Azetabulums. Der hintere Beckenring umfasst das Sakrum, Sakroiliakalgelenk, und das Ilium bis zum Azetabulum.

Eine Verletzung des vorderen Beckenringes ist für die biomechanische Stabilität des Beckenringes von untergeordneter Bedeutung (ca. 20\%).

Da die Kraftüberleitung des Stammskelettes von der Wirbelsäule über die Sakroiliakalgelenke, das Ilium und Azetabulum in die Beine verläuft. Eine Verletzung des hinteren Beckenringes dagegen bedeutet eine partielle oder komplette Unterbrechung dieses normalen Kraftflusses (ca. $80 \%$ ) mit entsprechend resultierender Instabilität des Beckenringes.

\section{Einteilung des Beckenringes in Frakturregionen}

Zur klinischen Beschreibung des betroffenen Frakturbereiches im ventralen und dorsalen Frakturbereich hat sich im täglichen Betrieb die Angabe der Frakturregion am Becken bewährt. Im vorderen Beckenring wird eine Symphysenruptur als transsymphysäre und Frakturen der Schambeinäste als transpubische Instabilitäten beschrieben. Im hinteren Beckenring können einerseits Luxationen der IS-Fuge als transiliosakrale Instabilitäten, Iliumfrakturen als transiliakale und Sakrumfrakturen als transsakrale Instabilitäten auftreten. Davon abzugrenzen sind die transazetabulären Instabilitäten, die als „Azetabulumfraktur“" gesondert betrachtet werden (Abb.4) $[2,13]$.

\section{Klassifikation der Beckenringfraktur}

M. E. Müller schrieb 1988 als Begleittext: „Eine Klassifikation ist nur dann nützlich, wenn sie die Schwere der Verletzung darstellt, als Basis für die Behandlung dient und später zum Vergleich der Resultate herangezogen werden kann“ [12]. In dieser These werden die Grundforderungen jeder Einteilung offenbar: Beschreibung der Verletzungsschwere durch Darstellung des Verletzungsmechanismus und des vorhandenen Instabilitätsgrades einer Fraktur. Aus der korrekten Einteilung soll dann konsekutiv die weitere Therapie abgeleitet werden können und durch Vergleich der Behandlungsergebnisse ergibt sich die resultierende Prognose für den Patienten.

\section{Entwicklung der Klassifikation}

Jede vorgestellte Klassifikation von Beckenringfrakturen spiegelt historisch betrachtet die diagnostischen und therapeutischen Möglichkeiten der jeweiligen Zeit wider. Malgaigne wies bereits 1847, also zu einer Zeit rein klinisch möglicher Diagnosen (Röntgenstrahlung erst ab 1895), auf die Bedeutung der Ringstruktur des Beckens hin und unterteilte in einfache und doppelte Ringunterbrechungen (sog. Malgaigne-Frakturen) [8]. Neben der Einteilung nach anatomischen Gesichtspunkten wurde in der Folgezeit vornehmlich nach dem Unfallmechanismus unterschieden [5, 6,7]. 1961 stellten Pennal und Sutherland ihre Klassifikation vor, die davon ausging, dass regelmäßig spezifische Unfallmechanismen zu drei Kraftrichtungen mit Beckenfrakturen führten, eine anterior-posteriore, eine laterale und eine vertikale „Scherverletzung“ [9]. In dieser Betrachtungsweise fehlte jedoch noch der Aspekt der verbleibenden Stabilität, um auch therapeutische Konsequenzen ziehen zu können. Tile ordnete 1991 den Unfallmechanismus dem Instabilitätsgrad unter, so dass nun eine Integration der Beckenringverletzung in das von der $\mathrm{AO}$ für sämtliche Frakturen verwendete $\mathrm{ABC}$ Schema möglich war. 1996 wurde die derzeit gebräuchliche CCF-Klassifikation der AO für das Becken vorgestellt (Nr. $61 \mathrm{Be}-$ cken, Beckenring), die die grundlegende Pennal-Einteilung mit der von Tile vorgeschlagenen Klassifikation verbindet und zur genauen Beschreibung und Analyse noch zusätzliche Qualifikatoren einführte [12]. Es werden zunächst Gruppen gebil$\operatorname{det}(A, B$ und $C)$, die jeweils durch Untergruppen weiter spezifiziert werden, um sämtliche mögliche Beckenfrakturen in einer numerischen Klassifikation unterscheiden zu können. Durch die Verwendung zusätzlicher Qualifikatoren können frakturspezifische Eigenschaften wie z.B. die Richtung der Krafteinwirkung, die Zahl der Fragmente oder die Lokalisation der Verletzung, beschrieben werden (Abb.5a-c). Vorteil dieser Klassifikation ist die hohe Zahl möglicher Kombinationen und der damit verbundenen wissenschaftlichen Vergleichbarkeit der Frakturtypen. Für den klinischen Gebrauch ist

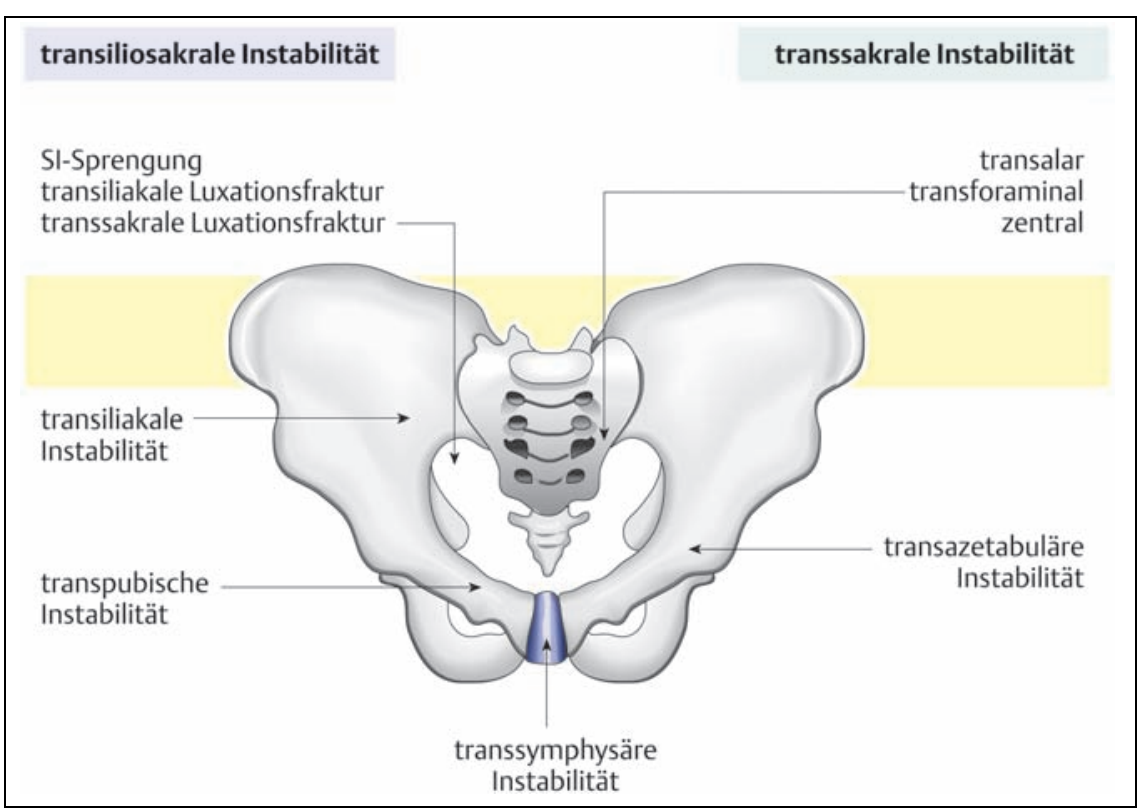

Abb. 4 Angabe der Instabilitätsrichtungen zur klinischen Beschreibung der Krafteinwirkungen und resultierenden Frakturverläufe am Becken. In Kombination mit dem Frakturtyp A, B oder C ergibt sich eine klinisch einfach anzuwendende Einteilung der Beckenringfraktur. 
eine solche alphanumerische Klassifikation jedoch gerade deshalb eher ungeeignet.

Zur Vereinfachung wird im täglichen klinischen Gebrauch nur die Gruppeneinteilung der Fraktur in Typ A, B oder C benutzt, um so zwischen stabilen und instabilen Frakturen unterscheiden zu können und zusätzlich wird eine Beschreibung der Verletzungsregion wie oben angeführt angegeben (Abb.4) [2,13].

\section{Typ-A-Fraktur}

Bei Typ-A-Frakturen bleibt die knöcherne und ligamentäre Integrität des dorsalen Beckenringes unangetastet.

Im Falle von knöchernen Abrissfrakturen, Beckenrand-, Scham- und Sitzbeinfrakturen, sowie Sakrumquerfrakturen distal der IS-Fuge wird die zentrale Kraftübertragung von der Wirbelsäule über die IS-Fuge auf beide Beine nicht kompromittiert, daher handelt sich um „stabile Frakturen".

\section{Typ-B-Fraktur}

B-Verletzungen führen zu einer partiellen Instabilität des Beckens.

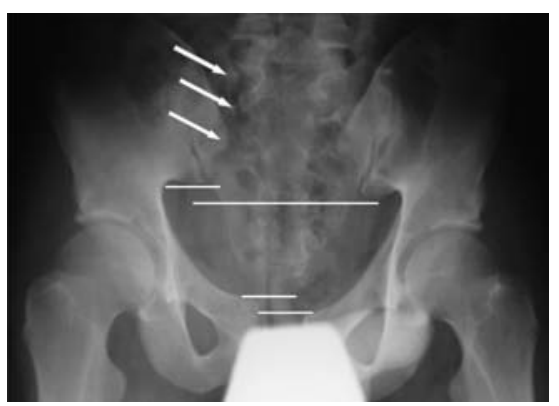

Abb. 5a Beispielhafte Darstellung eines Klassifikationsablaufes. Nach Anamneseerhebung vom erstbehandelnden Notarzt und primärer klinischer Untersuchung (fragliche Außenrotationsinstabilität re) wird eine primäre Beckenübersicht des 20-jährigen Motorradfahrers nach Verkehrsunfall vorgelegt. Die systematische Analyse des Bildes ergibt neben der transsymphysären Instabilität (Symphysenruptur) ventral eine transsakrale Instabilität dorsal. Die Fraktur scheint dabei in ihren oberen Anteilen transforaminal (s. Pfeile) und dann unterhalb S2 in die IS-Fuge auszulaufen, als Arbeitsdiagnose wird eine C-Verletzung angenommen, da ebenfalls ein Kranialversatz der rechten Beckenhälfte vorliegt (s. Querlinien). Bei bestehender Kreislaufstabilität wird für eine weitere Differenzierung eine $\mathrm{CT}$ des Beckens veranlasst (siehe $\mathbf{A} \mathbf{b} \mathbf{b} . \mathbf{5 b}$ ).
Die Gewalteinwirkung auf das Becken kommt entweder von lateral oder von medial meist auf die Beckenschaufel. Neben einer Instabilität im vorderen Bekkenring (z.B. Fraktur beider Schambeinäste oder einer Symphysensprengung) findet eine Mitverletzung der ventralen Bandstrukturen im Sakroiliakalgelenk statt. Entsteht die Verletzung durch ein scharnierartiges Aufklappen des Beckenringes, wird von einer Außenrotationsverletzung des Beckens gesprochen. Ein typisches Beispiel ist die so genannte „open book“-Verletzung des Beckenringes, bei der durch einen Außenrotationsmechanismus eine Symphysensprengung mit einer ventralen IS-Fugensprengung kombiniert ist. Das Pendant, eine Innenrotationsverletzung, entsteht durch einen lateralen Kompressionsmechanis- mus, im vorderen Beckenring kommt es zu einer Fraktur des oberen und unteren Schambeinastes und dorsalseitig zu einer Imprimierung des S1-Pedikels im ventralen Anteil. Bei einer beidseitigen Verletzung der SI-Fuge spricht man von einer bilateralen B-Fraktur.

\section{Typ-C-Verletzung}

C-Verletzungen sind gekennzeichnet durch eine komplette dreidimensionale Instabilität des Beckenringes, es findet eine komplette Unterbrechung der Ringstruktur statt.

Die verletzte Beckenhälfte ist nicht nur außen- oder innenrotiert, sondern es besteht auch eine zusätzliche Dislokation nach kranial oder kaudal. Die ursächliche

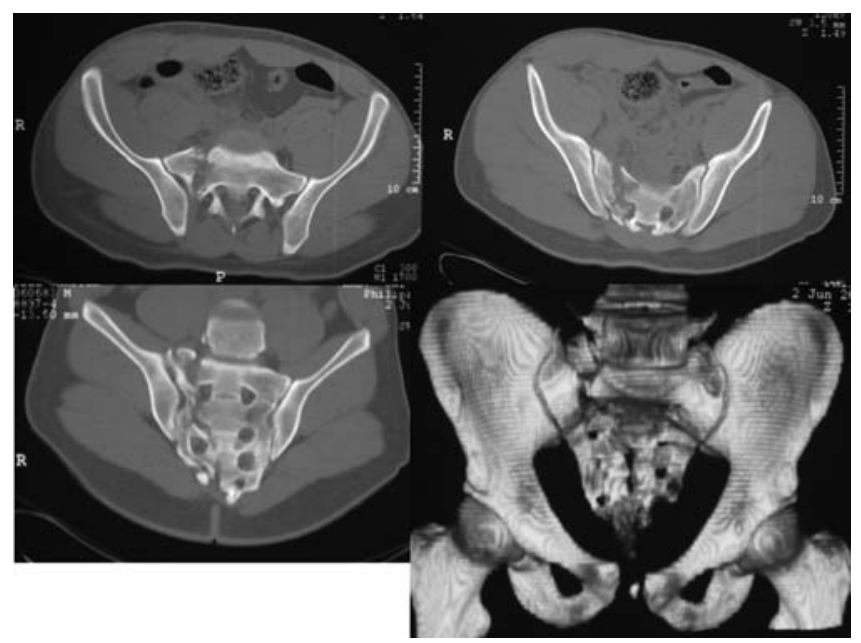

Abb.5b In der CT zeigt sich neben der klaffenden Symphysenruptur eine transforaminal verlaufende Sakrumfraktur mit Aussprengung zusätzlicher Fragmente.

\begin{tabular}{|c|c|c|c|}
\hline \multicolumn{4}{|c|}{ Frakturtyp C, Frakturgruppe 1 (nur unilateral), Fraktursubgruppe 1-3, Frakturuntergruppe 1-3 } \\
\hline Frakturtyp & $61-\mathrm{C}$ & $61-\mathrm{C}$ & $61-\mathrm{C}$ \\
\hline Frakturgruppe & $61-C_{1}$ & $61-C_{1}$ & $61-C_{1}$ \\
\hline Fraktursubgruppe & $\begin{array}{l}61-C 1.1 \\
\text { (llium) }\end{array}$ & $\begin{array}{l}61-\text { - C } 1.2 \\
\text { (SI-Gelenk) }\end{array}$ & $\begin{array}{r}61-\text { C } 1.3 \\
\text { (Sakrum) }\end{array}$ \\
\hline Frakturuntergruppe & $\begin{array}{l}61-C 1.1 .1 \\
\text { keine }\end{array}$ & $\begin{array}{l}61-\text { C } 1.2 .1 \\
\text { (transiliakale Lux.Fx) } \\
61-\text { C } 1.2 .2 \\
\text { (reine SI-Sprengung) } \\
61-\text { C } 1.2 .3 \\
\text { (transsakrale Lux.Fx) }\end{array}$ & $\begin{array}{l}61-C 1.3 .1 \\
\text { (transalar) } \\
61-C 1.3 .2 \\
\text { (transforaminal) } \\
61-C 1.3 .3 \\
\text { (zentral) }\end{array}$ \\
\hline \multicolumn{4}{|l|}{ Modifikator C } \\
\hline \multicolumn{4}{|c|}{ Modifikator C (anteriore Verletzung): } \\
\hline \multicolumn{3}{|c|}{ 1: unilateral, transpubisch, ipsilateral } & , aberlappend \\
\hline \multicolumn{2}{|c|}{ 2: unilateral, transpubisch, contralateral } & 7: Symphysensp & + transpubisch ipsilateral \\
\hline \multicolumn{3}{|c|}{ 3: bilateral, transpubisch } & + transpubisch contralateral \\
\hline \multicolumn{3}{|c|}{ 4: Symphsensprengung, $\$ 2,5 \mathrm{~cm}$} & + transpubisch bilateral \\
\hline \multicolumn{3}{|c|}{ 5: Symphysensprengung $>2,5 \mathrm{~cm}$} & tzung \\
\hline
\end{tabular}

Abb.5c Mit den gesammelten Informationen aus Beckenübersicht und den CT-Schnitten sowie den 2D und 3D-Reformationen kann die Fraktur eindeutig nach oben abgebildeten Schema klassifiziert werden: Frakturtyp C, Frakturgruppe C-1, Fraktursubgruppe C-1.3 Sakrum, Frakturuntergruppe C-1.3.2 transforaminal. Die Symphysensprengung wird als Modifikator C mit der Nr. 4 aufgefunden. Resultierendes Ergebnis der Klassifikation: AO 61 C 1.3.2 (Modifikator C 4). 
Krafteinleitung kann hierbei aus allen Richtungen kommen. Durch die komplette Unterbrechung der Ringstruktur handelt es sich definitionsgemäß um eine instabile Fraktur (Abb.5a-c). Zur Wiederherstellung der Stabilität ist eine operative Versorgung notwendig.

\section{Sakroiliakalgelenk}

Im Sakroiliakalgelenk selbst finden nur geringe Bewegungen (Nutation) durch die äußerst straffen Bandzüge des Gelenkes statt. Insbesondere die dorsalen Bandanteile verbinden das Sakrum hängebrückenartig mit dem Ilium und verhindern ein Herausrutschen des Sakrums nach ventral. Die Bänder reichen dabei anatomisch auf der Sakrumrückfläche bis an die Neuroforamina heran. Eine Verletzung des SI-Gelenkes kann aus einer Luxation oder Luxationsfraktur (transiliakal, transsakral) bestehen. Die Luxation der SI-Fuge kann die ventralen Bandanteile (Ligg. sacroiliacalia ventralia), die im Gelenk befindlichen interossären Bänder oder die besonders straffen hinteren Bänder umfassen. Bei Zerreißung aller drei Bandanteile des Gelenkes liegt eine instabile Luxation des SI-Gelenkes vor, es handelt sich somit um eine Typ-C-Verletzung. Kommt es dagegen z.B. durch einen Außenrotationsmechanismus nur $\mathrm{zu}$ einer Zerreißung der ventralen Bandanteile liegt eine rotationsinstabile Typ-B-Verletzung wegen der erhaltenen dorsalen Bandzüge vor. Die Luxationsfrakturen beginnen häufig im Sakrum oder Ilium und verlaufen dann in Höhe des zweiten Sakralwirbels in das SI-Gelenk mit konsekutiver Zerreißung der Bandstrukturen hinein. Die zumeist durch einen Innenrotationsmechanismus entstehenden transsakralen Instabilitäten (Impressionsfraktur im Sakrum) stellen infolge der dorsalen Überbrückung der Fraktur durch die Bandzüge nur partiell instabile B-Verletzungen dar und führen in der Regel nicht zu einer kranio-kaudalen Versetzung des dorsalen Beckenringes.

\section{Sakrum}

Aufgrund seiner zentralen Positionierung im hinteren Beckenring und als Verbindungsglied zwischen Wirbelsäule und Becken werden Verletzungen des Sakrums gesondert klassifiziert.

Nach Denis et al. werden am Sakrum drei Zonen unterschieden, durch die Frakturen typischerweise verlaufen; die transalare, die transforaminale und die zentrale Zone [3].

Transalare Frakturverläufe kommen zwar prozentual am häufigsten vor (50\%), werden aber durch die biomechanisch wichtigen dorsalen Sakroiliakalbänder überbrückt und somit stabilisiert. Ebenso sind die seltenen zentralen Sakrumfrakturen (15\%) aufgrund des spongiösen Frakturverlaufes biomechanisch stabil. Von klinischer Bedeutung sind die relativ häufigen transforaminalen Sakrumfrakturen (34\%), da sie nicht nur zu einer erheblichen Instabilität im dorsalen Beckenring führen, sondern auch mit einer hohen Rate neurologischer Schädigungen vergesellschaftet sind (28\%) [3].

\section{Schlussfolgerung}

Der einheitliche Gebrauch der Terminologie bei Beckenringfrakturen führt $\mathrm{zu}$ einer gemeinsamen und verständlichen Sprache in Diagnostik und Bewertung der Verletzung. Einheitliche Definitionen helfen, die Verletzungsschwere richtig zu beurteilen und stellen damit wichtige therapeutische Weichen.

Durch Einführung einer numerischen Klassifikation nach dem ABC-Schema wurde eine eindeutige Beschreibung der einwirkenden Kraft, der vorliegenden Frakturregion und der verbleibenden Stabilität des Beckenringes möglich. Eine korrekte Anwendung dieser Klassifikation ermöglicht somit nicht nur den Vergleich der einzelnen Frakturtypen miteinander, sondern gibt auch durch die Angabe der verbleibenden Beckenringstabilität den Ausschlag für die einzuleitende Therapie und beeinflusst so die Behandlungsprognose entscheidend mit.

\section{Literatur}

${ }^{1}$ Bosch U, Pohlemann T, Haas N, Tscherne H. Klassifikation und Management des komplexen Beckentraumas. Unfallchirurg 1992; 96: 189-196

${ }^{2}$ Culemann U, Reilmann H. Verletzungen des Beckenringes. Unfallchirurg 1997; 100 (6): 487-496

${ }^{3}$ Denis F, Steven D, Comfort T. Sacral fractures: An important problem. Retrospective analysis of 236 cases. Clin Orthop 1988. 227: $67-81$

${ }^{4}$ Gilliland M, Ward R, Barton R, Miller P, Duke J. Factors affecting mortality in pelvic fractures. J Trauma 1982; 22: 691 -93

${ }^{5}$ Isler B, Ganz R. Klassifikation der Beckenringverletzung. Unfallchirurg 1990; 93: 289-302

${ }^{6}$ Helfet D. Alpha-numeric classifications for pelvic fractures. In: Surgery of the pelvis and Acetabulum: an international consensus. Conference proceeding, Pittsburgh (1992)

${ }^{7}$ Huittinen V, Slätis P. Fractures of the pelvis:trauma mechnism, types of injury and principles of treatment. Acta Chir Scand 1972; 138: $563-569$

${ }^{8}$ Malgaigne J. Traites des fractures et des luxationes. Balliere, Paris (1847)

${ }^{9}$ Pennal G, Sutherland G: Fractures of the pelvis (motion picture). Amercan Academy of Orthopedic surgeons (AAOS) Film library, Park Tidge II, (1961)

${ }^{10}$ Pohlemann T, Tscherne H, Baumgärtel F, Egbers HJ, Euler E, Maurer T, Fell M, Mayr E, Quirini W, Schlickewei W, Weinberg A. Beckenverletzungen: Epidemiologie, Therapie und Langzeitverlauf. Unfallchirurg 1996; 99: 160-167

${ }^{11}$ Rothenberger D, Fischer R, Strate R, Valesco $\mathrm{R}$, Perry J. The mortality associated with pelvic fractures. Surgery 1978; 84 (No.3): 356 59

${ }^{12}$ Tile M, Helfet D, Kellam J, Isler B, Nazarian S, Müller ME. Comprehensive Classification of fractures. M E Müller Foundation, Bern 1996; No.61: 1 -6

${ }^{13}$ Tscherne H, Pohlemann T. (Hrsg.) Becken und Acetabulum, Springer Verlag, Berlin Heidelberg New York 1998

${ }^{14}$ Wilker D, Izbicki J, Euler E, Schweiberer L. Verletzungen des Beckens und der Retroperitonealorgane. Urologe 1991; 30: $183-$ 88

\section{Dr. med. Ulf Culemann}

Facharzt für Chirurgie - Unfallchirurgie

Chirurgische Universitätsklinik

Abt. für Unfall-, Hand- und Wiederherstellungschirurgie

Universitätskliniken des Saarlandes

Kirrberger Str.

D-66421 Homburg/Saar

Prof. Dr. med. Heinrich Reilmann Chefarzt der Unfallchirurgischen Klinik

Städtisches Klinikum Braunschweig Unfallchirurgische Klinik

Städtisches Klinikum Braunschweig

Holwedestr. 16

D-38118 Braunschweig 